aided by the loading of heavier calcareous and ferruginous deep-sea deposits. Since the principal elevation and erosion of the continental border strips fronting the Atlantic took place during the Palæozoic, it is suggested that the present conformation of the Atlantic bottom dates back in part at least to that era.

\title{
THE IONIZATION AND ACTIVITY OF LARGELY IONIZED SUBSTANCES
}

\author{
By Arthur A. Noyes and Duncan A. Macinnes \\ Research laboratory of Physical, Chemistry, Massachusetrs institute \\ OF TECHNOLOGY
}

Read before the Academy, November 10, 1919

In applications of the ionic theory of solutions it is customary to employ, in accordance with the original hypothesis of Arrhenius, as a measure of the degree of ionization of salts, acids, and bases, the ratio of the equivalent conductance of the substance at any given concentration to the limiting value of the equivalent conductance as the concentration approaches zero, where the ionization may be assumed to be complete.

This assumption, however, is not a necessary conclusion from the fundamental theory of ionic conduction. According to this theory, the equivalent conductance $\Lambda$ (which is by definition the quantity of electricity which under a potential-difference of one volt passes per second between electrodes of indefinite extent $1 \mathrm{~cm}$. apart, between which is placed that quantity of solution which contains one equivalent weight of the ionizing substance) is for a uniunivalent substance given by the expression $\Lambda=\gamma \mathrm{F}\left(\mathrm{U}^{+}+\mathrm{U}^{-}\right)$, in which $\gamma$ is the fraction of the substance ionized (equal in this case to the number of equivalents of each ion present in the solution), $\mathrm{F}$ the quantity of electricity (96,500 coulombs) associated with each equivalent of ion, and $\mathrm{U}^{+}$and $\mathrm{U}^{-}$the mobilities of the positive and negative ions (that is, their velocities through the solution under a potential-gradient of one volt per centimeter). For the equivalent conductance $\Lambda_{\circ}$ at zero concentration, where the ionization becomes complete, we have the corresponding expression $\Lambda_{0}=F\left(U_{0}{ }^{+}+U_{0}{ }^{-}\right)$. Combining these two equations we get

$$
\frac{\Lambda}{\Lambda_{0}}=\gamma \frac{\mathrm{U}^{+}+\mathrm{U}^{-}}{\mathrm{U}_{0^{+}}+\mathrm{U}_{\mathrm{o}}^{-}}
$$

From this equation it is evident that $\Lambda / \Lambda_{\circ}$ is equal to the ionization only when the mobilities of the ions can be assumed constant up to the concenvtration under consideration. That they should remain constant so long as the solution does not differ appreciably from water as a viscous medium, may seem reasonable; but, in view of possible electrical effects resulting from the large electric charges on the ions, it is by no means certain. 
This simple assumption has been justified in the case of slightly ionized acids and bases, where the ion-concentration in the solution is small, by the fact that the so-determined ionization values change with the concentration just as the mass-action law requires. This, however, is not true even approximately in the case of salts and of the largely ionized acids and bases (such as hydrochloric acid and sodium hydroxide); and we are forced to conclude, either that owing to the change in the ion-mobilities the conductance-ratio is not a correct measure of ionization, or that the chemical activity or mass-action effect of ions, and perhaps also of the unionized molecules present with them, is not proportional to their concentration, as the ordinary mass-action law assumes.

In either case the conductance-ratio affords us no reliable information as to the chemical activity of ions; and we must turn to other properties for a quantitative measure of this important factor, which determines the equilibrium of all chemical reactions between salts, acids, and bases in solution, and also the magnitude of many physical properties thermodynamically related to the activities.

The term activity must, in the first place, be defined in a precise way; and from a chemical standpoint the most practical method is to define it, as was proposed by $\mathrm{G}$. N. Lewis, ${ }^{1}$ as the quantity which when substituted for the concentration of the substance in mass-action expressions will express its effect in determining the equilibrium. Thus the activity of the substance is its "effective concentration" from this mass-action view-point.

Correspondingly, the most obvious method of determining the relative activities of a substance in solutions of different concentrations is to find its concentrations in a gaseous phase in equilibrium with the solutions; for in gases at low pressure the concentration and activity can ordinarily be assumed to be proportional. For example, we know that the ratio of the activities of un-ionized hydrochloric acid in its 11-molal and 8-molal aqueous solution at $30^{\circ}$ is 12.0 , since the partial vapor-pressures of the hydrochloric acid in those solutions have been found to be 11.3 and 0.94 $\mathrm{mm}$. of mercury. This quantity is also the ratio of the products of the activities of the hydrogen-ion and chloride-ion in the two solutions, since these products are by definition the quantities that must be substituted in the mass-action expression for the equilibrium of the reaction $\mathrm{HCl}=$ $\mathrm{H}^{+}+\mathrm{Cl}^{-}$. Thus denoting the pressures in the two solutions by $p_{1}$ and $p_{2}$, the activities of the un-ionized molecules by $a_{1}$ and $a_{2}$, and those of the ions by $a_{1}^{+}, a_{1}^{-}$, and $a_{2}{ }^{+}, a_{2}^{-}$, we get:

$$
\frac{p_{1}}{p_{2}}=\frac{a_{1}}{a_{2}}=\frac{a_{1}+a_{1}^{-}}{a_{2}+a_{2}^{-}}
$$

This direct method is, however, of very limited applicability in the case of largely ionized substances, since they seldom have appreciable vapor- 
pressures. We have recourse, therefore, to a simple thermodynamic relation between activity and electromotive force. This relation may be derived by considering the work or free-energy decrease attending the transfer of one mol of the substance (for example, of $1 \mathrm{HCl}$ ) from the solution in which its vapor-pressure is $p_{1}$ to that in which it is $p_{2}$. This freeenergy decrease $-\Delta F$ is given by the familiar expression $-\Delta F=R T$ $\log \left(p_{1} / p_{2}\right)$. In this expression, in view of the above considerations, we may substitute for the vapor-pressure ratio the ratio $\left(a_{1}+a_{1}^{-}\right) /\left(a_{2}+a_{2}^{-}\right)$of the product of the activities of the ions, yielding the equation

$$
-\Delta F=R T \log \frac{a_{1}+a_{1}^{-}}{a_{2}+a_{2}-}
$$

We thus obtain what may be regarded as a secondary, but more general definition or measure of activity.

The simplest process from a theoretical standpoint (aside from that already described involving passage through the vapor phase) by which a substance can be transferred from one solution to another is one in which this transfer is brought about in a voltaic cell. Thus in the case of hydrochloric acid, we can cause a transfer of one mol of the acid from concentration $c_{1}$ (activity $a_{1}$ ) to concentration $c_{2}$ (activity $a_{2}$ ) by causing one faraday ( $F$ coulombs) to pass through the cell

$$
\mathrm{H}_{2} \text { (1 atm.), } \mathrm{HCl}\left(c_{2}\right), \mathrm{AgCl}+\mathrm{Ag}, \mathrm{HCl}\left(c_{1}\right), \mathrm{H}_{2} \text { (1 atm.). }
$$

The electromotive force $\mathrm{E}$ of this cell multiplied by the quantity of electricity $F$ is, therefore, the work which can be obtained from the change in state under consideration, or the free-energy change attending it. That is,

$$
-\Delta F=\mathrm{EF}=R T \log \frac{a_{1}+a_{1}^{-}}{a_{2}{ }^{+} a_{1}^{-}}=R T \log \frac{c_{1}^{2} \alpha_{1}+\alpha_{1}^{-}}{c_{2}^{2} \alpha_{2}{ }^{+} \alpha_{2}^{-}} .
$$

It will be noted that in the last of these expressions there has been written, in place of the activities $a$ of the ions, products $c \alpha$, in which the quantity $\alpha$, called the activity-coefficient, is evidently the factor by which the total concentration $c$ of the substance must be multiplied to give the activity of the ion.

By the use of this electromotive-force method the authors of this paper have, with the aid of their students, carried out a series of determinations of the activities of typical substances. Two of these researches, those on potassium chloride ${ }^{2}$ and on hydrochloric acid, ${ }^{3}$ have already been published; and two more, on lithium chloride and potassium hydroxide, will soon be described in detail in the Journal of the American Chemical Society. These last investigations were carried out, with the aid of grants from the Carnegie Institution of Washington, by Mr. J. A. Beattie and Mr. Ming Chow, respectively, using cells, with dropping amalgam electrodes, of the following types: 


$$
\begin{aligned}
& \mathrm{Ag}+\mathrm{AgCl}, \mathrm{LiCl}\left(c_{2}\right), \mathrm{Li} \text { in } \mathrm{Hg}, \mathrm{LiCl}\left(c_{1}\right), \mathrm{AgCl}+\mathrm{Ag} . \\
& \mathrm{Hg}+\mathrm{HgO}, \mathrm{KOH}\left(c_{2}\right), \mathrm{K} \text { in } \mathrm{Hg}, \mathrm{KOH}\left(c_{1}\right), \mathrm{HgO}+\mathrm{Hg} .
\end{aligned}
$$

It is the purpose of this paper to summarize and compare the results of these investigations, and to state the general conclusions to which they lead.

From the observed values of the electromotive forces of these cells there were calculated by the equation given above the products $\alpha^{+} \alpha^{-}$of the activity-coefficients of the two ions at various concentrations, the value of the products at the lowest concentration $(0.001$ or 0.0035 molal $)$ at which accurate measurements could be made being assumed equal to the conductance-ratio $\Lambda / \Lambda_{0}$ at that concentration. These activity-coefficient products were plotted against the logarithm of the concentrations, and those corresponding to round concentrations were read off.

The following table contains the values of the square root of the soobtained products, that is, the values of the expression $\left(\alpha^{+} \alpha^{-}\right)^{1 / 2}$, which represents the geometrical mean of the activity-coefficients of the positive and negative ions of the substance. This table also contains the corresponding values of the conductance-ratio $\Lambda / \Lambda_{\circ}$, multiplied by the ratio $\eta / \eta_{\circ}$ of the viscosity of the solution to that of pure water, this last serving to correct approximately for the frictional resistance of the medium to the passage of the ions through it.

TABLE 1

\begin{tabular}{|c|c|c|c|c|c|c|c|c|}
\hline \multirow{2}{*}{$\begin{array}{l}\text { Mols. per } \\
1000 \mathrm{~g} \text {. } \\
\text { water }\end{array}$} & \multicolumn{4}{|c|}{ ACTIVITY-COEFFICIENTS } & \multicolumn{4}{|c|}{ CONDUCTANCE-VISCOSITY RATIOS } \\
\hline & $\mathrm{KCl}$ & $\mathrm{LiCl}$ & $\mathrm{HCl}$ & KOH & $\mathrm{KCl}$ & $\mathrm{LiCl}$ & $\mathrm{HCl}$ & KOH \\
\hline 0.001 & 0.979 & 0.976 & & & 0.979 & 0.976 & 0.990 & \\
\hline 0.003 & 0.943 & 0.945 & 0.990 & 0.982 & 0.968 & 0.962 & 0.986 & 0.980 \\
\hline 0.005 & 0.923 & 0.930 & 0.965 & 0.975 & 0.956 & 0.949 & 0.981 & 0.975 \\
\hline 0.010 & 0.890 & 0.905 & 0.932 & 0.961 & 0.941 & 0.932 & 0.972 & 0.963 \\
\hline 0.030 & 0.823 & 0.848 & 0.880 & 0.920 & 0.914 & 0.904 & 0.957 & 0.939 \\
\hline 0.050 & 0.790 & 0.817 & 0.855 & 0.891 & 0.889 & 0.878 & 0.944 & 0.925 \\
\hline 0.100 & 0.745 & 0.779 & 0.823 & 0.846 & 0.860 & 0.846 & 0.925 & 0.910 \\
\hline 0.200 & 0.700 & 0.750 & 0.796 & 0.793 & 0.827 & 0.812 & 0.909 & 0.891 \\
\hline 0.300 & 0.673 & 0.738 & 0.783 & 0.769 & 0.807 & 0.792 & 0.903 & 0.889 \\
\hline 0.500 & 0.638 & 0.731 & 0.773 & 0.765 & 0.779 & 0.766 & 0.890 & 0.884 \\
\hline 0.700 & 0.618 & 0.734 & 0.789 & 0.772 & 0.761 & 0.751 & 0.874 & 0.879 \\
\hline 1.000 & 0.593 & 0.752 & 0.829 & 0.786 & 0.742 & 0.737 & 0.845 & 0.877 \\
\hline 2.000 & & & 1.040 & & & & & \\
\hline 3.000 & & 1.164 & 1.402 & & & & & \\
\hline
\end{tabular}

Activity-Coefficients and Conductance-Viscosity Ratios

The table contains the results of only the above-described researches carried out under our direction. It should be mentioned, however, that exact electromotive-force measurements from which activities can be derived have also been made by $\mathrm{Jahn}^{4}$ on potassium, sodium, and hy- 
drogen chlorides, by Harned ${ }^{5}$ on potassium chloride, and by Linhart ${ }^{6}$ on hydrochloric acid. As the results of Harned supplement at higher concentrations those for potassium chloride here presented, it may be stated that, assuming a constant transference-number of 0.497 for the potassium ion and an activity-coefficient of 0.745 at 0.1 molal as given in the table, his data lead to the following values:

$\begin{array}{llllllll}\text { Normal concentration. } \ldots \ldots \ldots \ldots & 0.2 & 0.3 & 0.5 & 0.7 & 1.0 & 2.0 & 3.0 \\ \text { Activity-coefficient. . . . } \ldots \ldots \ldots & 0.688 & 0.657 & 0.624 & 0.608 & 0.593 & 0.572 & 0.586\end{array}$

The results presented in the table may be summarized as follows:

1. In the case of all four substances the activity-coefficient decreases with increasing concentration much more rapidly than does the conductance-viscosity ratio, the differences amounting to from 7 to $15 \%$ at 0.1 molal, and from 5 to $18 \%$ at 0.5 molal.

2. In the case of all the substances except potassium chloride the activity-coefficient, unlike the conductance-viscosity ratio, passes through a pronounced minimum in the neighborhood of 0.5 molal, afterwards increasing rapidly at the higher concentrations. Even potassium chloride, according to Harned's data, has a minimum activity-coefficient in the neighborhood of 2-normal.

3. The activity-coefficient even at moderate concentrations varies considerably with the nature of the substance; thus its value at 0.5 molal is $64 \%$ for potassium chloride, $73 \%$ for lithium chloride, and $77 \%$ for hydrochloric acid and for potassium hydroxide.

From these facts we may draw the general conclusions that the conductance ratio can no longer be regarded as even an approximate measure of the activity of the ions of largely ionized substances in their mass-action and thermodynamic relations; that this activity varies with the concentration, differently in the case of different substances; and that for the present it can be determined only empirically for each substance, with the aid of measurements of chemical equilibria, electromotive force or freezingpoint. ${ }^{7}$

It is, moreover, evident that the activity-coefficient of the ion-constituents cannot be proportional to and mainly determined by the fraction of the substance ionized, for this fraction could not increase with increasing concentration unless the ionizing power of the medium becomes much greater at moderate concentrations; and even then, it could not become greater than unity, as is actually the case with the activity-coefficient of hydrochloric acid above 2 -molal.

The results here presented do not show whether or not the conductanceratio is equal to the degree of ionization. But these two quantities can hardly be equal in view of the fact that the conductances of the two ionconstituents of most uniunivalent substances seem to vary by different percentage amounts with increasing concentration, as may be seen from 
Noyes and Falk's ${ }^{8}$ summary of the experimentally determined transference-numbers. These numbers, which are equal to the ratio $\mathrm{U}^{+} / \mathrm{Cu}^{+}+$ $\mathrm{U}^{-}$) of the mobility of the cation-constituent to the sum of the mobilities of the two ion-constituents, show variations that correspond to changes in the ratio $\mathrm{U}^{+} / \mathrm{U}^{-}$of the mobilities of the two ion-constituents between zero concentration and 0.3 normal, of $41 / 2 \%$ in the case of sodium chloride, $7 \frac{1}{2} \%$ in the case of hydrochloric acid, and $24 \%$ in the case of lithium chloride. Moreover, MacInnes ${ }^{9}$ has shown that the chloride-ion constituent has the same equivalent conductance in 0.1 normal solutions of lithium chloride, potassium chloride, and hydrochloric acid, although the conductance-ratios $\Lambda / \Lambda_{\circ}$ for these substances are $0.833,0.862$ and 0.925 ; so that, if we account for the constancy of the chloride-ion conductance by the probable assumptions that the three substances are equally ionized and that the chloride-ion has the same mobility in the three solutions, we must conclude that the hydrogen-ion and lithium-ion decrease in mobilities between zero concentration and 0.1 normal by amounts that differ from each other by $10 \%$ - a result that makes it not unreasonable to suppose that the whole decrease in equivalent conductance (of $71 / 2 \%$ and $16 \%$ in the two cases) may be due entirely to decrease in mobility of the ions, and not at all to decrease in ionization.

When, indeed, in addition to these conclusions that neither the activitycoefficients nor the conductance-ratio is determined primarily by the degree of ionization, we take into consideration the fact that there is no property which affords any direct evidence of the existence of un-ionized molecules in solutions of most of the largely ionized inorganic substances up to moderate concentrations, it seems advisable to adopt for the present the hypothesis that such substances are completely ionized, and to attribute the decrease in the conductance-ratio wholly to decrease of ionmobility, and the change in activity-coefficient entirely to some unknown effect of a physical nature.

It would lead far beyond the scope of this paper to discuss the many classes of phenomena that seem to substantiate this assumption. A summarized description of them was given many years ago by one of the authors of this paper, ${ }^{10}$ who at that time, however, suggested that they might be explained more fully by the hypothesis that the ions are partially united as a result solely of their electrical attraction into loosely bound molecules, which differ fundamentally from the stable molecules formed as a result of chemical affinity in accordance with the law of mass-action. The known facts may, however, prove to be better accounted for by the simple hypothesis of complete ionization, supplemented by some other, purely physical, explanation of the cause of the decrease of ion-mobility and of ion-activity with increasing concentration; and this now seems the most promising method of treatment, as has recently been urged by various authors. ${ }^{11}$ As said above, we cannot here discuss in detail the hypothesis 
of complete ionization; but in closing it may be pointed out that it accounts for the remarkable facts that so many very dissimilar chemical substances (for example, hydrochloric acid and potassium chloride) seem to be equally ionized, and that a volatile substance like hydrochloric acid does not have an appreciable vapor-pressure even in 1-normal solution where $15 \%$ of it must be assumed to be in the un-ionized state, if the conductance-ratio is taken as a measure of ionization. It may also be mentioned that it avoids the improbable conclusions as to the abnormal activity of the un-ionized molecules to which solubility-effects, interpreted under the older assumptions, lead. ${ }^{12}$

${ }^{1}$ Lewis, Proc. Amer. Acad., 43, 1907 (259-293); Zs. physik. Chem., 61, 1908 (129-165).

2 MacInnes and Parker, J. Amer. Chem. Soc., 37, 1915 (1445-1461).

${ }^{3}$ Ellis, Ibid., 38, 1916 (737-762); Noyes and Ellis, Ibid., 39, 1917 (2532-2544).

4 Jahn, Zs. physik. Chem., 33, 1900 (559-576).

5 Harned, J. Amer. Chem. Soc., 38, 1916 (1989).

${ }^{6}$ Linhart, Ibid., 4I, 1919 (1175-1180).

${ }^{7}$ Lewis, Ibid., 34, 1912 (1635); Bates, Ibid., 37, 1915 (1421-1445).

8 Noyes and Falk, Ibid., 33, 1911 (1454).

- MacInnes, Ibid., 4I, 1919 (1086).

${ }^{10}$ Noyes, "The Physical Properties of Aqueous Salt Solutions in Relation to the Ionic Theory," Congress of Arts and Sciences, St. Louis Exposition, 4, 1904 (317); Science, 20, 1904 (582); abstract, Zs. physik. Chem., 52 (635); also Noyes, J. Amer. Chem. Soc., 30, 1908 (335-353).

11 Milner, Phil. Mag., 35, 1918 (214, 354); Ghosh, Trans. Chem. Soc. (London), I I3, 1918 (449, 627); Bjerrum, Zs. Elektrochem., 24, 1918 (321).

12 Bray, J. Amer. Chem. Soc., 33, 1911 (1673-1686).

THE COMMUTATIVITY OF ONE-PARAMETER TRANSFORMATIONS IN REAL VARIABLES

By ARTHur C. LUNN

Department of Mathematics, University of Chicago

Communicated by E. H. Moore, November 10, 1919

If $X=\Sigma \xi_{i}\left(x_{1} \ldots x_{n}\right) \frac{\partial}{\partial x_{i}}$ and $Y=\Sigma \eta_{i}\left(x_{1} \ldots x_{n}\right) \frac{\partial}{\partial x_{i}}$ be the differential symbols of two one-parameter transformations, the known condition for the fcommutativity of the finite transformations is the identical vanishing of the commutator $(X Y)=X Y-Y X$. The proof given by Lie-Engel, applying to the case of analytic functions, depends on expansions in powers of the canonical parameters. The computation of $(X Y)$ according to the original definition involves the use of the second partial derivatiyes of the coefficients $\xi, \eta$, but the final form, containing only first derivatives, is 